\title{
Interleukin 32 expression in human melanoma
}

\author{
Helicia Paz ${ }^{1+}{ }^{0}$, Jennifer Tsoi ${ }^{3,5+}$, Anusha Kalbasi ${ }^{1,4,6}$, Catherine S. Grasso ${ }^{5}$, William H. McBride ${ }^{4}$, \\ Dörthe Schaue ${ }^{4}$, Lisa H. Butterfield ${ }^{7,8,9,10}$, Deena M. Maurer ${ }^{7}$, Antoni Ribas ${ }^{1,5,6}$, Thomas G. Graeber \\ and James S. Economou $1,2,3,6^{*}$
}

\begin{abstract}
Background: Various proinflammatory cytokines can be detected within the melanoma tumor microenvironment. Interleukin 32 (IL32) is produced by T cells, NK cells and monocytes/macrophages, but also by a subset of melanoma cells. We sought to better understand the biology of IL32 in human melanoma.

Methods: We analyzed RNA sequencing data from 53 in-house established human melanoma cell lines and 479 melanoma tumors from The Cancer Genome Atlas dataset. We evaluated global gene expression patterns associated with IL32 expression. We also evaluated the impact of proinflammatory molecules TNFa and IFNY on IL32 expression and dedifferentiation in melanoma cell lines in vitro. In order to study the transcriptional regulation of IL32 in these cell lines, we cloned up to $10.5 \mathrm{~kb}$ of the $5^{\prime}$ upstream region of the human IL32 gene into a luciferase reporter vector.

Results: A significant proportion of established human melanoma cell lines express IL32, with its expression being highly correlated with a dedifferentiation genetic signature (high AXL/low MITF). Non IL32-expressing differentiated melanoma cell lines exposed to TNFa or IFN $\gamma$ can be induced to express the three predominant isoforms $(\alpha, \beta, \gamma)$ of IL32. Cis-acting elements within this $5^{\prime}$ upstream region of the human IL32 gene appear to govern both induced and constitutive gene expression. In the tumor microenvironment, IL32 expression is highly correlated with genes related to T cell infiltration, and also positively correlates with high AXL/low MITF dedifferentiated gene signature.
\end{abstract}

Conclusions: Expression of IL32 in human melanoma can be induced by TNFa or IFNY and correlates with a treatment-resistant dedifferentiated genetic signature. Constitutive and induced expression are regulated, in part, by cisacting sequences within the $5^{\prime}$ upstream region.

Keywords: Interleukin 32 (IL32), Melanoma dedifferentiation, IL32 transcriptional regulation, Immune infiltration, Myeloid polarization

\section{Background}

Though first described in 1992 as NK4, interleukin 32 (IL32) [1, 2] was recharacterized in 2005 as a proinflammatory cytokine differentially expressed in IL18 responsive cells. Since then, its expression has been implicated in various pathologies, including rheumatoid arthritis, pathogen responses, atherosclerosis, and several malignancies [3-12]. IL32 is broadly expressed in immune

\footnotetext{
*Correspondence: jeconomou@mednet.ucla.edu

†Helicia Paz and Jennifer Tsoi are co-first authors

1 Department of Surgery, University of California, Los Angeles, 10833 Le

Conte Ave, Los Angeles, CA 90095, USA

Full list of author information is available at the end of the article
}

cells, including natural killer cells [13-15], T lymphocytes [16-18], macrophages, and dendritic cells $[19,20]$. Functionally, IL32 can activate NF-kB and p38 mitogen activated protein kinase pathways and induce expression of proinflammatory cytokines including TNF $\alpha$, IL8 and CCL2. In addition to its presence in immune tissues, IL32 expression can also be induced in human epithelial tissues. IL32 is also expressed in a broad range of human epithelial cancers-gastric [3], lung [5], breast [4], colon [6-9], pancreas [10], and thyroid [11, 12]-as well as hematologic malignancies such as lymphoma and leukemia $[16,21]$. IL32 expression in cancer is linked to 
features associated with worse prognosis, including angiogenesis, invasion, and metastasis [4].

However, the role of IL32 in human melanoma cells is less well understood. Initial studies suggested that IL32 expression was related to a more invasive, metastatic phenotype marked by a loss of e-cadherin expression [22]. Others reported that IL32 isoforms alpha and gamma were highly enriched in PD-L1 expressing melanoma specimens [23].

Herein we report an unbiased analysis of IL32 expression in melanoma using RNA sequencing data from: [1] a large in-house panel of melanoma cell lines and [2] tumor tissues from The Cancer Genome Atlas (TCGA) dataset. IL32 expression correlated with a dedifferentiated phenotype which has been characterized by resistance to targeted therapies, escape from immune recognition, and a high AXL/low MITF genetic signature [24-27]. Expression of IL32 in human melanoma can be transcriptionally regulated in the context of a proinflammatory tumor microenvironment with either induced or constitutive IL32 expression strongly, but not invariably, correlating with a dedifferentiated genetic signature.

\section{Methods}

\section{Cell culture}

The human melanoma cell lines were established from patient biopsies. These cells were grown in RPMI 1640 media supplemented with $10 \% \mathrm{FBS}, 1 \% \mathrm{~L}$-glutamine and $1 \%$ penicillin/streptomycin/actinomycin D (Life Technologies, Grand Island, NY). Melanoma cell lines were treated with $1000 \mathrm{IU} / \mathrm{mL}$ TNF $\alpha$ and $100 \mathrm{IU} / \mathrm{mL}$ IFNY (PeproTech US, Rocky Hill, NJ) for the indicated time points. M397 was cultured with recombinant $100 \mathrm{ng} /$ mL IL32 $\alpha,-\beta$, or $-\gamma$ (R\&D Systems, Minneapolis, MN) for 7 days, with fresh IL32 being added every $2-3$ days. Cells were counted using Trypan blue. Jurkat $\mathrm{T}$ lymphocytes were cultured in RPMI 1640 supplemented with 10\% FBS.

\section{Gene expression analysis}

Gene expression FPKM values for the melanoma cell line panel was obtained from the Gene Expression Omnibus (GEO) accession number GSE80829. The hallmark geneset for NF- $\mathrm{kB}$ and TNF $\alpha$ signaling (TNFA_SIGNALING_VIA_NFKB) was downloaded from the Molecular Signatures Database (MSigDB). Gene set enrichment analysis of the full ranked list of IL32 correlations was performed using the pre-ranked tool and the GO Biological Process Ontology gene sets from MSigDB v6.1. Gene expression FPKM values of skin cutaneous melanoma tumor biopsies from The Cancer Genome Atlas (TCGA SKCM) was downloaded from the Genomic Data Commons (portal.gdc.cancer.gov). For all analyses, FPKM values were $\log 2$ transformed with an offset of 1 . Calculation of the enrichment overlap of the top 100 correlated genes with IL32 was performed using the MSigDB investigate gene sets tool (software.broadinstitute.org/gsea/ msigdb/annotate.jsp) and the hallmark gene sets.

\section{Retroviral vectors}

IL32 isoforms were cloned into an MSCV retroviral vector with a T2A-GFP using the HiFi DNA Assembly Cloning Kit (NEB, Ipswich, MA). The retroviral vector was kindly provided by A. Ribas. Retroviruses were produced using GP2-293 cells (Clontech, Mountain View, CA) and human melanoma cells were transduced with IL32 expressing retroviral vectors with $4 \mathrm{ug} / \mathrm{mL}$ Polybrene. GFP positive cells were sorted $48 \mathrm{~h}$ after transduction. Cells maintained approximately $95 \%$ GFP positivity over the course of the experiment. IL32 expression was confirmed by real time PCR and Western blot.

\section{Real time PCR}

RNA was extracted using PureLink RNA Mini Kit (ThermoFisher, Waltham, MA) and cDNA was synthesized using the High-Capacity RNA-to-cDNA kit (ThermoFisher, Waltham, MA). Real time PCRs were performed using the Applied Biosystems 7500 using the following primers designed using NCBI Primer-BLAST. GAPDH was used a housekeeping gene.

IL32 Primers:

IL32a Fwd: 5'-GAGGCAACAGATCCCCTGTC-3'. IL32a Rev: $5^{\prime}$-GGCTCCGTAGGACTTGTCAC-3'. IL32b Fwd: 5'-TCTCTCGGCTGAGTATTTGTGC-3'. IL32b Rev: 5'-ATGACCCAGCTCCACTGAGA-3'. IL32 g Fwd: 5'-TACTTCTGCTCAGGGGTTGG-3'. IL32 g Rev: 5'-TGGGTGCTGCTCCTCATAAT-3'. Differentiation gene set:

NGFR Fwd: 5'-TCATCCCTGTCTATTGCTCCA-3'. NGFR Rev: 5'-TGTTCTGCTTGCAGCTGTTC-3'. MLANA Fwd: 5'-GCTCATCGGCTGTTGGTATT- ${ }^{\prime}$. MLANA Rev: $3^{\prime}$-TTCTTGTGGGCATCTTCTTG-5'. MITF Fwd: $5^{\prime}$-ATCAGCAACTCCTGTCCAGC- $3^{\prime}$. MITF Rev: $5^{\prime}$-GCCAGTGCTCTTGCTTCAGA-3'. AXL Fwd: 5'-ACCTACTCTGGCTCCAGGATG-3'. AXL Rev: 5'-CGCAGGAGAAAGAGGATGTC- $3^{\prime}$. GAPDH Fwd: 5'-TGCACCACCAACTGCTTAGC-3'. GAPDH Rev: 5'-GGCATGGACTGTGGTCATGAG-3'.

\section{$5^{\prime}$ rapid amplification of CDNA ends (RACE)}

To determine the transcriptional start site of IL32, 5' RACE was performed according the manufacturer's directions for the SMARTer RACE $5^{\prime} / 3^{\prime}$ kit (Takara, Mountain View, CA). Briefly, RNA was isolated from the IL32 expressing cell line, M318, using NucleoSpin RNA II kits (Takara, Mountain View, CA). First strand cDNA 
synthesis was done according the manufacturer's directions. The $5^{\prime}$ RACE reaction using the following gene specific primer and a universal primer (Universal Primer Short, UPM, Takara) using 25 cycles of $94^{\circ}$ for $30 \mathrm{~s}, 68^{\circ}$ for $30 \mathrm{~s}$, and $72^{\circ}$ for $3 \mathrm{~min}$. RACE products were run on an agarose gel and purified using the NucleoSpin Gel and PCR Clean-Up kit (Takara, Mountain View, CA). RACE products were cloned into the pRACE vector using the In-Fusion HD Cloning kit (Takara, Mountain View, CA) and sequenced using the M13F and M13R sequencing primers.

\section{Luciferase constructs}

The promoter region of IL32 were pcr'd from the Human BAC clone RP11 (RPC1-11 clone 473M20) using Pfusion DNA polymerase (NEB, Ipswich, MA). Promoter region greater than $3 \mathrm{~kb}$ were cloned into pGL3 using HiFi DNA Assembly Cloning Kit (NEB, Ipswich, MA). The promoter regions were derived using the following primers.

$-10.5 \mathrm{~kb}$ Fwd: 5'-ACCGAGCTCTTACGCGTGCTA GCCCGCAGGGAAGAAGGTGAGAGATG-3'.

$-7.5 \mathrm{~kb}$ Fwd: 5'-ACCGAGCTCTTACGCGTGCTA GCCCCTCTTTAGCGGTGAGTGGGG-3'.

$-5.5 \mathrm{~kb}$ Fwd: 5'-ACCGAGCTCTTACGCGTGCTA GCCCCAGTAGCTGGCTGTTTCGTG-3'.

$-2.5 \mathrm{~kb}$ Fwd: 5'-CTAGCCCGGGCTCGAGATCTA GAAAGTAGATGAGGCCAG-3'.

-2 kb Fwd: 5'-CTAGCCCGGGCTCGAGATCTAAAA CAGGGTACATACAGTCTG- $3^{\prime}$.

$-1.5 \mathrm{~kb}$ Fwd: 5'-CTAGCCCGGGCTCGAGATCTG GAGTGCAGTGGCACCAT- 3 '.

$-1.0 \mathrm{~kb}$ Fwd: 5'-CTAGCCCGGGCTCGAGATCTG TTTGGCCCCAGGAAACC- 3 '.

$-0.5 \mathrm{~kb}$ Fwd: 5'-CTAGCCCGGGCTCGAGATCTC CCCAGCCAGCTGTCCCG- $3^{\prime}$.

$-2.5 \mathrm{~kb}$ to $-0.5 \mathrm{~kb}$ Rev: $5^{\prime}$-CTCGAGCTCGAGTGG CGGCCAAAAGTTCAAGGAGC- $3^{\prime}$.

$-10.5 \mathrm{~kb}$ to $-3 \mathrm{~kb}$ Rev: 5'-GAATGCCAAGCTTAC TTAGATCGCATGGCGGCCAAAAGTTCAAG- ${ }^{\prime}$.

Promoter regions were cloned into the SmaI/XhoI (less than $-3.0 \mathrm{~kb}$ ) or HindIII (greater than $-3.0 \mathrm{~kb}$ ) sites of pGL3 Luciferase reporter vector (Promega, Madison, WI). Promoter constructs were confirmed by Sanger sequencing. Promoter constructs were transfected into $1.8-2 \times 10^{4}$ cells human melanoma cells in equal molar concentrations using Lipofectamine LTX with PLUS Reagent (ThermoFisher, Waltham, MA). pGL4.74 (hRluc/TK, Promega, Madison, WI) was used for transfection normalization. Firefly and Renilla luciferase expression was assessed $48 \mathrm{~h}$ after transfection using the Dual-Glo Luciferase Assay system (Promega, Madison, WI). Cells were treated with $1000 \mathrm{IU} / \mathrm{mL}$ TNF $\alpha$ and $100 \mathrm{IU} / \mathrm{mL}$ IFN $\gamma$ (PeproTech US, Rocky Hill, NJ) 24 h prior to assessing luciferase activity. All values were normalized to the pGL3 empty vector control.

\section{Myeloid cell polarization}

PBMCs were collected using a ficoll gradient (Ficoll-Paque Plus, GE Healthcare) and monocytes were isolated by a CD14 positive selection (Miltenyi, Bergisch Gladbach, Germany). Monocytes were split into five experimental groups: (1) Negative Control (no cytokines), (2) GM-CSF (Sanofi) + IL-4 (Cell Genix) at $1000 \mathrm{U} / \mathrm{ml}$, 3) Recombinant IL32 $\alpha$ (R\&D Systems) at $100 \mathrm{ng} / \mathrm{ml}, 4)$ Recombinant IL32 $\beta$ (R\&D Systems) at $100 \mathrm{ng} / \mathrm{ml}, 5$ ) Recombinant IL32 $\gamma$ (R\&D Systems) at $100 \mathrm{ng} / \mathrm{ml}$, and cultured using Cell Genix Media to yield immature DCs at day 5. Immature DC were harvested and surface stained for flow cytometry analysis. Cell surface markers were observed on the double positive, HLA-DR and CD86 population of cells. Antibodies used included CD80 FITC (BD, Clone L307.4), Mouse IgG1 FITC (Beckman Coulter PN IM0639U), CD86 Pe-Cy7 (BD, Clone FUN-1), HLA-DR PerCpCy5.5 (BD, Clone G46-4), CD1B APC (BioLegend, Clone SN13), CD14 APC-Cy 7 (BD, Clone MфP9), CD68 BV 711 (BD, Clone Y1/82A), Mouse IgG2B BV 711 (BD, Clone 27-35), and Zombie Aqua Viability Dye BV 510 (BioLegend).

\section{Statistical analyses}

Gene expression bar graphs are shown as mean and standard differentiation. Gene expression was normalized to GAPDH and expressed as Delta Ct values. Comparison between the reference control (untreated M397, M398, and M249) was performed with one-way analysis of variance (ANOVA, 95\% CI) with a Dunnetts' multiple comparisons test. All calculations were done using Prism software. p-values less than 0.05 were considered statistically significant. For luciferase activity, data was normalized to Renilla luciferase expression and fold changes were calculated against the pGL3 empty control vector. Error bars represent standard deviation.

\section{Results}

\section{IL32 expression in human melanoma}

We examined RNA sequencing data from a panel of 53 established human melanoma cell lines derived from resected metastatic deposits $[25,26]$. Across this dataset, IL32 expression was detectable in a majority of the melanoma cell lines (Fig. 1a). IL32 expression in a subset of melanoma cell lines was confirmed by real time PCR (data not shown). Immunohistochemical staining of IL32 in melanoma tissues, pulled from The Human Protein Atlas, indicates a range of expression within tumor samples (Additional file 1: Figure S1A) [28, 29]. However, it is notable that IL32 levels in melanoma are low relative to other tumor cell lines represented in the 


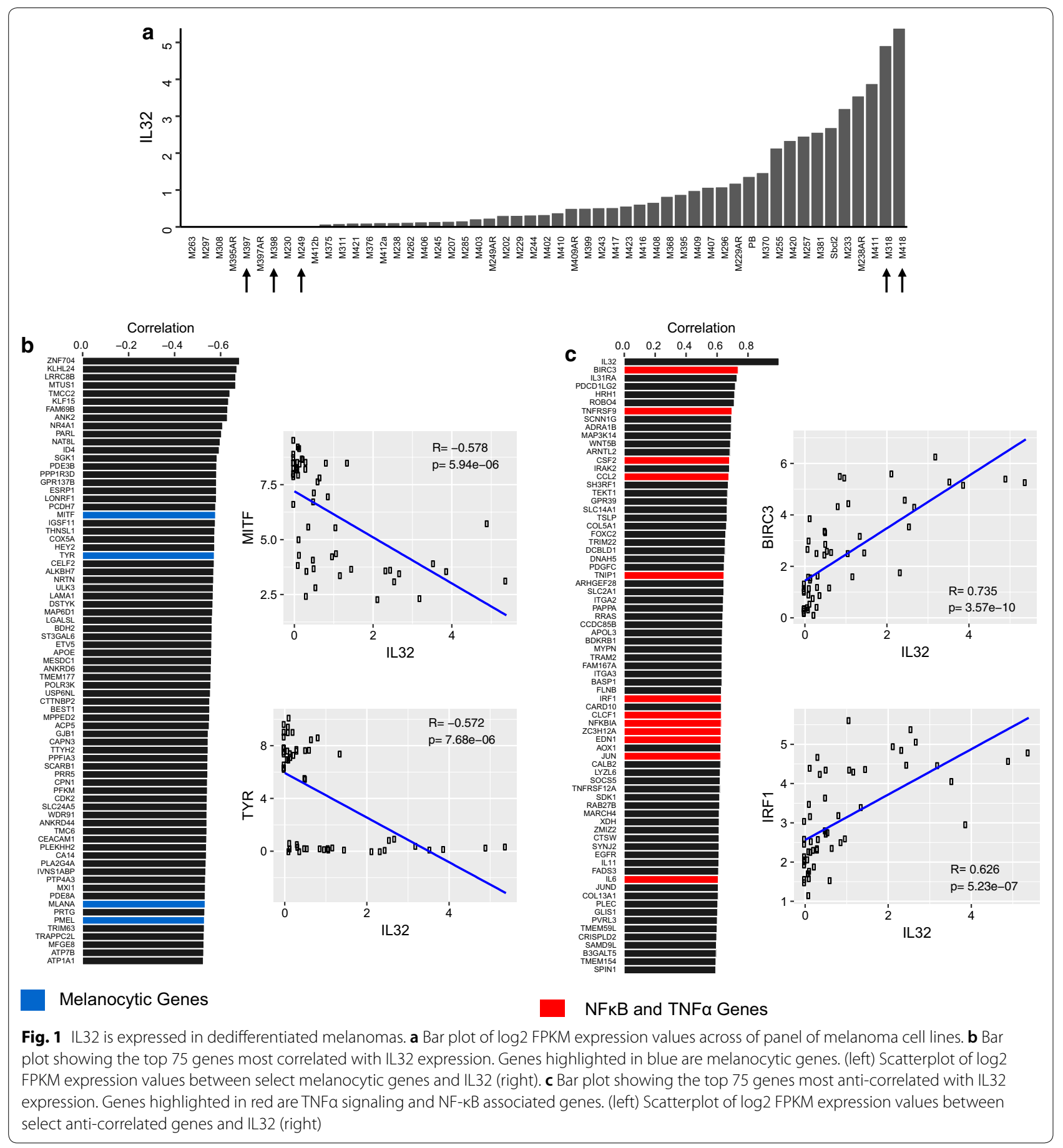

NCI-60 Cancer Cell Line database or the Cancer Cell Line Encyclopedia (Additional file 1: Figures S1B, C, respectively) [30, 31].

\section{IL32 expression in human melanoma cell lines and tissues correlates with a dedifferentiated gene signature}

We globally assessed IL32 co-expression patterns across the 53 cell lines by calculating the Pearson correlation between the expression of IL32 to all genes in the dataset. Among the top 75 anti-correlated genes with IL32 expression, we found classical melanocyte and pigment associated genes such as MITF, TYR, PMEL, and MLANA (Fig. 1b). Gene expression scatterplots of MITF and TYR vs. IL32 show that melanoma cells with high IL32 expression are typically dedifferentiated, whereas differentiated melanoma are associated with absent or lower levels of 
IL32 (Fig. 1b). Furthermore, among the top 20 differentially expressed genes in melanoma cell lines in the lowest quartile of IL32 expression (compared to top quartile), we identified enrichment of genes related to pigmentation and melanogenesis (TYR, MLANA, PMEL, TYRP1, $D C T$, and MITF; hypergeometric p-value $=7.9 \times 10^{-15}$ ).

Previous studies have shown that a dedifferentiated MITF-low transcriptional state is associated with an NF-kB-high inflammatory cell state and that TNF $\alpha$ treatment can promote dedifferentiation [24, 27, 32]. Therefore, we investigated if genes positively correlated to IL32 were involved in NF- $\mathrm{B}$ or TNF $\alpha$ signaling. To assess this, we obtained NF- $\mathrm{B}$ a and TNF $\alpha$ signaling genes from the well-curated hallmark collection [33]. Within the top 75 genes highly correlated to IL32 expression, we observed a strong enrichment for the NF- $\mathrm{kB}$ and TNF $\alpha$ hallmark genes (hypergeometric $\mathrm{p}$-value $=2.1 \times 10^{-11}$ ) (Fig. 1c). Scatterplots for representative genes are shown in Fig. 1c. TNF $\alpha$ and IL32 interactions have previously been described in the literature [16].

As a confirmatory step, we performed Gene Set Enrichment Analysis on the full ranked list of IL32 expression correlations. Supportive of our earlier findings, the top gene sets highly enriched for IL32 anti-correlation include pigmentation and metabolic gene sets, and the top most highly enriched for IL32 expression include $\mathrm{NF}-\kappa \mathrm{B}$ and immune related gene sets (Tables 1, 2). Together, this suggests that IL32 is among several hundred melanoma genes whose expression is affected by exposure to these proinflammatory cytokines.

\section{TNFa and IFN $\gamma$ induced IL32 expression in general synchrony with dedifferentiation in melanoma cell lines}

To evaluate if IL32 is induced with inflammatory signaling, three non-IL32-producing melanoma cell lines (M397, M398, M249) were exposed to TNF $\alpha$ or IFNy for $72 \mathrm{~h}$ (Fig. 2). We selected these three melanoma cell

Table 1 Gene set enrichment analysis (GSEA) of genes correlated with IL32

\begin{tabular}{|c|c|c|c|}
\hline Name & NES & p-value & $\begin{array}{l}\text { FDR } \\
\text { q-value }\end{array}$ \\
\hline 1. Go I kappab kinase NF kappab signaling & 2.461 & $<0.001$ & $<0.001$ \\
\hline 2. Go cellular response to mechanical stimulus & 2.456 & $<0.001$ & $<0.001$ \\
\hline 3. Go positive regulation of chemotaxis & 2.302 & $<0.001$ & $2.008 \mathrm{E}-03$ \\
\hline 4. Go tumor necrosis factor mediated signaling pathway & 2.286 & $<0.001$ & $1.506 \mathrm{E}-03$ \\
\hline 5. Go modification by symbiont of host morphology or physiology & 2.266 & $<0.001$ & $1.449 E-03$ \\
\hline 6. Go response to mechanical stimulus & 2.257 & $<0.001$ & $1.601 \mathrm{E}-03$ \\
\hline 7. Go positive regulation of response to external stimulus & 2.223 & $<0.001$ & $2.745 E-03$ \\
\hline 8. Go toll like receptor signaling pathway & 2.217 & $<0.001$ & $2.698 \mathrm{E}-03$ \\
\hline 9. Go pattern recognition receptor signaling pathway & 2.215 & $<0.001$ & $2.533 \mathrm{E}-03$ \\
\hline 10. Go NIK NF kappab signaling & 2.212 & $<0.001$ & $2.280 \mathrm{E}-03$ \\
\hline
\end{tabular}

Top ten enriched gene sets correlated with IL32 include NF-KB and immune-related gene sets

Table 2 Gene set enrichment analysis (GSEA) of genes anti-correlated with IL32

\begin{tabular}{|c|c|c|c|}
\hline Name & NES & p-value & $\begin{array}{l}\text { FDR } \\
\text { q-value }\end{array}$ \\
\hline 1. Go developmental pigmentation & -2.619 & $<0.001$ & $<0.001$ \\
\hline 2. Go melanocyte differentiation & -2.500 & $<0.001$ & $<0.001$ \\
\hline 3. Go cellular respiration & -2.456 & $<0.001$ & $<0.001$ \\
\hline 4. Go mitochondrial respiratory chain complex assembly & -2.446 & $<0.001$ & $<0.001$ \\
\hline 5. Go oxidative phosphorylation & -2.400 & $<0.001$ & $<0.001$ \\
\hline 6. Go pigmentation & -2.377 & $<0.001$ & $<0.001$ \\
\hline 7. Go pigment cell differentiation & -2.374 & $<0.001$ & $<0.001$ \\
\hline 8. Go aerobic respiration & -2.347 & $<0.001$ & $1.83 E-04$ \\
\hline 9. Go tricarboxylic acid metabolic process & -2.251 & $<0.001$ & $6.51 \mathrm{E}-04$ \\
\hline 10. Go mitochondrial respiratory chain complex I biogenesis & -2.228 & $<0.001$ & 8.04E-04 \\
\hline
\end{tabular}

Top ten enriched gene sets negatively correlated with IL32 include pigmentation and metabolic gene sets 
lines to observe the parallel impact of TNF $\alpha$ and IFN $\gamma$ on IL32 and the melanoma differentiation state because they exhibited a low AXL/high MITF differentiated genetic signature. Both TNF $\alpha$ and IFN $\gamma$ induced expression of the three predominant isoforms of IL32 $(\alpha, \beta, \gamma)$ in all three cell lines at varying levels. Overall, the impact of TNF $\alpha$ on IL32 expression was greater than the impact of IFN $\gamma$. Induction of IL32 $\gamma$ by TNF $\alpha$ was the most notable response in all three cell lines. The relative level of IL32 isoform transcripts in these three cell lines, after TNFo induction, is generally lower than the constitutive expression in the two high IL32-producing, dedifferentiated cell lines M318 and M418 (Fig. 3a). For reference, we also evaluated IL32 transcript levels in the Jurkat T cell line, a recognized high IL32 expressing leukemia cell line. In general, with the exception of M318, the $\gamma$ isoform is the most abundant and when induced by cytokine stimulation has significantly higher and more prolonged induction (Figs. 2, 3a, b).

To test the parallel impact of IL32 induction with $\mathrm{TNF} \alpha$ or IFN $\gamma$ treatment on differentiation, we also evaluated expression of melanocytic markers MITF and
MLANA, neural crest marker NGFR and the receptor tyrosine kinase $A X L$. A large and growing body of literature has shown the expression of AXL strongly inversely correlated with the expression of MITF, with a high AXL/ MITF ratio associated with therapy resistance [24, 25]. At baseline, these three melanoma cell lines exhibited a low AXL/high MITF differentiated genetic signature. In two of three cell lines (M397 and M398) we observed inversion of the AXL/MITF ratio towards a dedifferentiated genotype in parallel with induction of IL32. However, one of three cell lines (M249) did not dedifferentiate in response to TNF $\alpha$ or IFN $\gamma$, despite induction of IL32. The lack of correlation between IL32 induction and inverted AXL/MITF ratio in M249 suggests that one event is not invariably accompanied by the other. Furthermore, enforced expression of recombinant IL32 $\alpha,-\beta$, or $-\gamma$ in M397 did not impact expression of melanoma differentiation genes MITF, AXL, NGFR or MLANA, strongly suggesting that IL32 is not a causal or upstream event in the melanoma dedifferentiation process (Additional file 2: Figure S2).

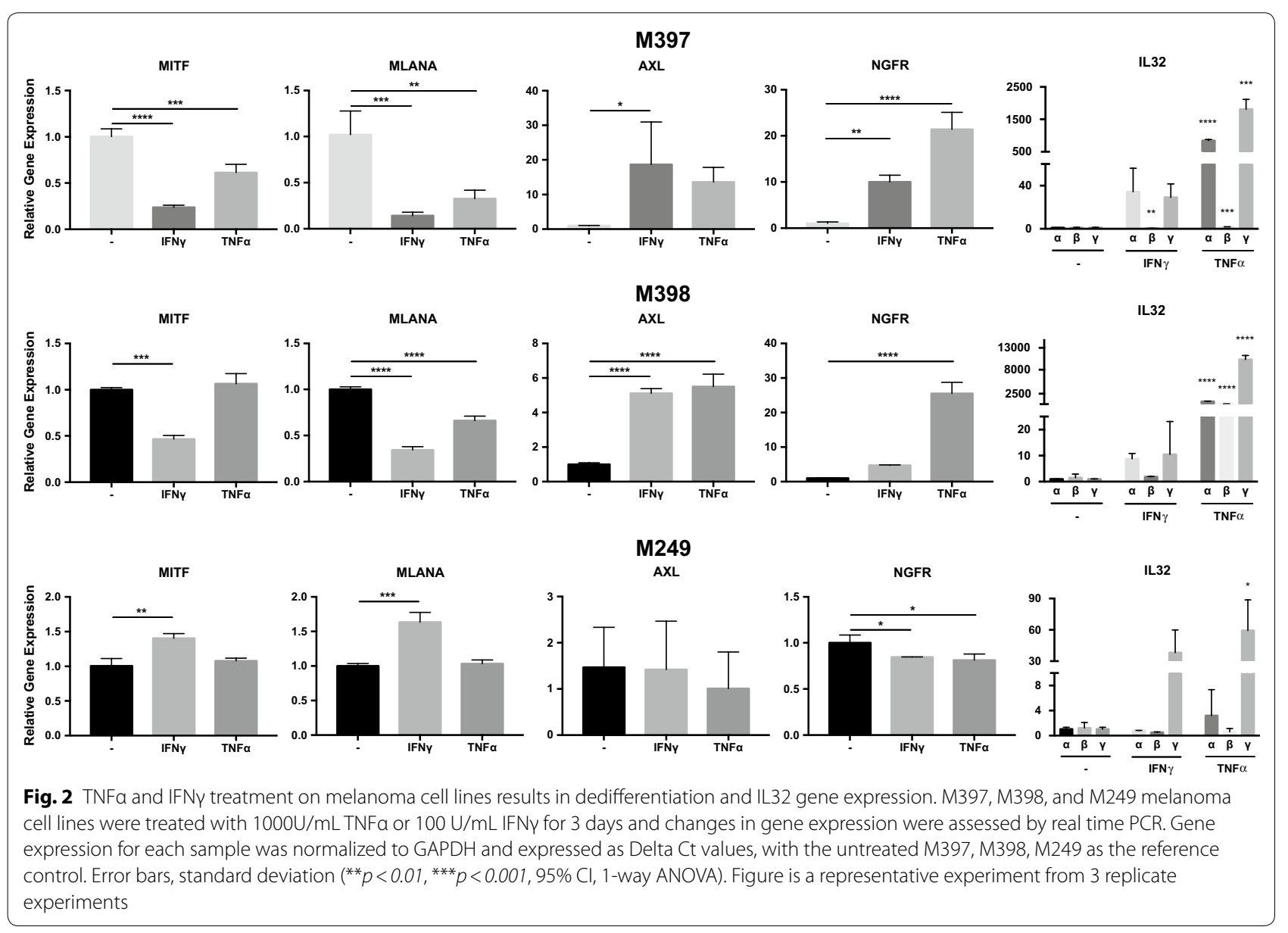



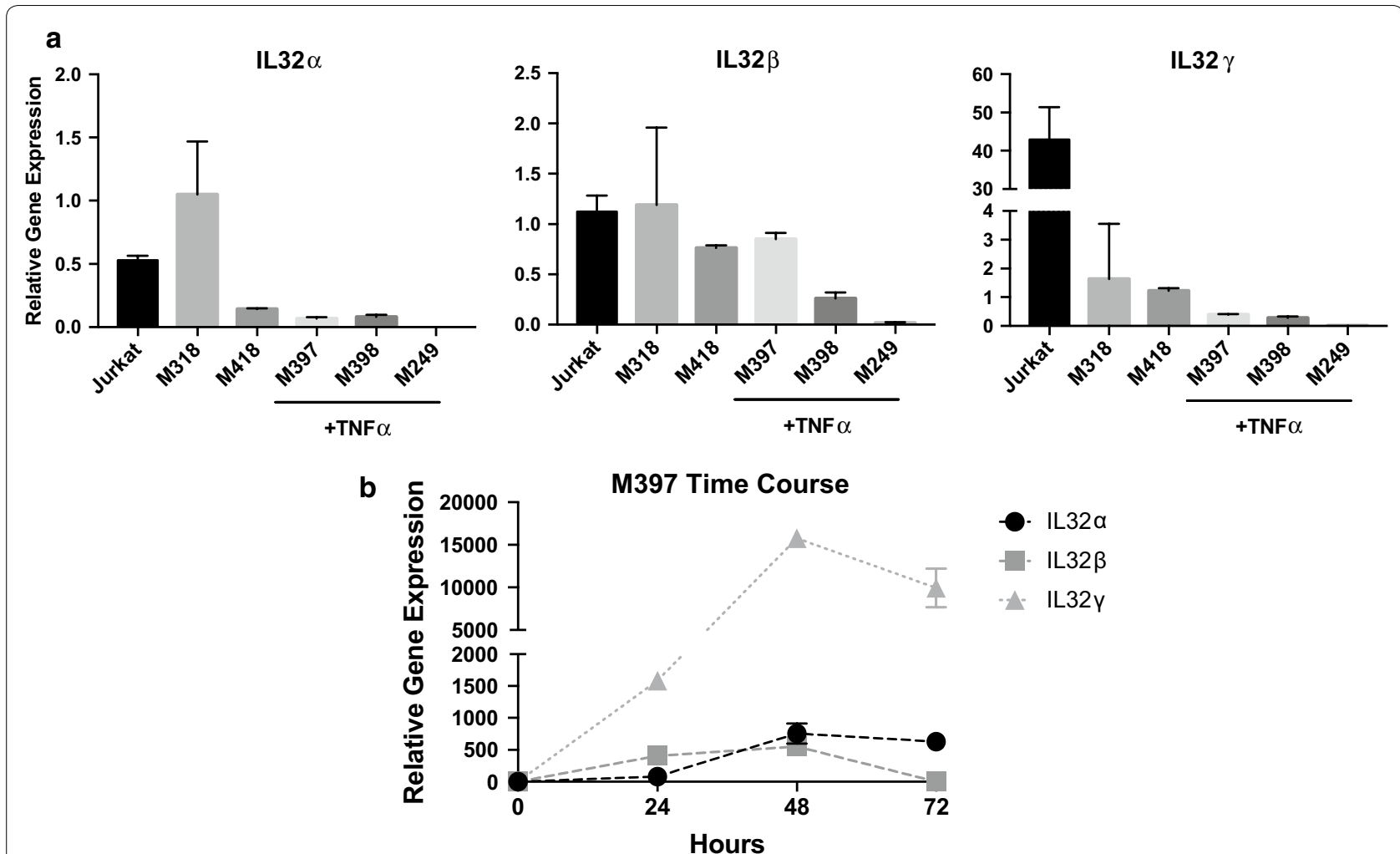

Fig. 3 Level of IL32 isoform expression after TNFa treatment. a IL32 gene expression $(\alpha, \beta, \gamma)$ after 3 day treatment with $1000 \mathrm{U} / \mathrm{mL}$ TNFa was compared to two IL32 expressing melanoma cell lines (M318 and M418) and Jurkat cells. Gene expression was normalized to GAPDH and expressed as Delta Ct values, with the Jurkat cells as the reference control. b Induction of IL32 gene expression with 24, 48, and $72 \mathrm{~h}$ treatment with $1000 \mathrm{U} /$ $\mathrm{mL}$ TNFa. Gene expression was normalized to GAPDH and Delta Ct values are compared to the day 0 reference control

\section{Proinflammatory cytokines TNFa and IFN $\gamma$ induce IL32 promoter activity in melanoma cell lines}

We characterized the transcriptional regulation of IL32 in human melanoma, both in constitutive and cytokineinduced cells. CHIP-seq analysis of active enhancer and promoter elements, as defined by H3K4Me1, H3K27Ac, H3K3Me3 marks [34-37] in HUVEC and K562, cell lines known to express IL32, indicate the presence of regulatory elements $5^{\prime}$ relative to the IL32 translational start site (ATG) (Fig. 4a, ENCODE data set; University of California, Santa Cruz (UCSC) genome browser). Using $5^{\prime}$ RACE we determined the location of the transcription start site (TSS) in the IL32 positive cell line M318; two TSS were identified at $-464 \mathrm{bp}$ and -175 bp (chr16: 3,115,349 and chr16: 3,115,638) relative to the ATG (Fig. 4b). The Eukaryotic Promoter Database (EPD; http://epd.vital.it.ch) identified two putative IL32 promoters EPD NK4_1 and EPD IL32_1, which are $-558 \mathrm{bps}$ and $-221 \mathrm{bps}$ relative to the ATG (see "Discussion" Fig. 4b). Based on this information, we cloned various lengths of the IL32 $5^{\prime}$ upstream genomic region $(-10.5,-7.5,-5.5,-2.5,-2,-1.5$, $-1,-0.5)$ into a luciferase reporter construct; these constructs encompassed the putative EPD promoters, both identified TSS sites, and the upstream $\left(5^{\prime}\right)$ ENCODE histone marks. These constructs were transiently transfected into IL32 negative (M249, M397) and IL32 positive (M318) cell lines (Fig. 4d). Strong promoter activity was noted in the IL32 positive but not the negative cell lines, with the highest level in the -10.5 and -1.5 constructs. When both IL32 negative cell lines were exposed to TNF $\alpha$ or IFN $\gamma$ for $24 \mathrm{~h}$, there was a significant induction of promoter activity. TNF $\alpha$ consistently induced stronger IL32 promoter activity than IFN $\gamma$. M249, which cannot be dedifferentiated with TNF $\alpha$ or IFN $\gamma$, also showed strong IL32 promoter induction consistent with IL32 transcript expression. The promoter construct profiles were similar in both constitutive and induced settings.

\section{IL32 in the melanoma microenvironment is associated} with immune infiltration and melanoma dedifferentiation Given the impact of TNF $\alpha$ and IFN $\gamma$ on IL32 expression in melanoma cell lines, we investigated the expression of IL32 in the chemokine-rich melanoma tumor microenvironment using RNA sequencing data from 


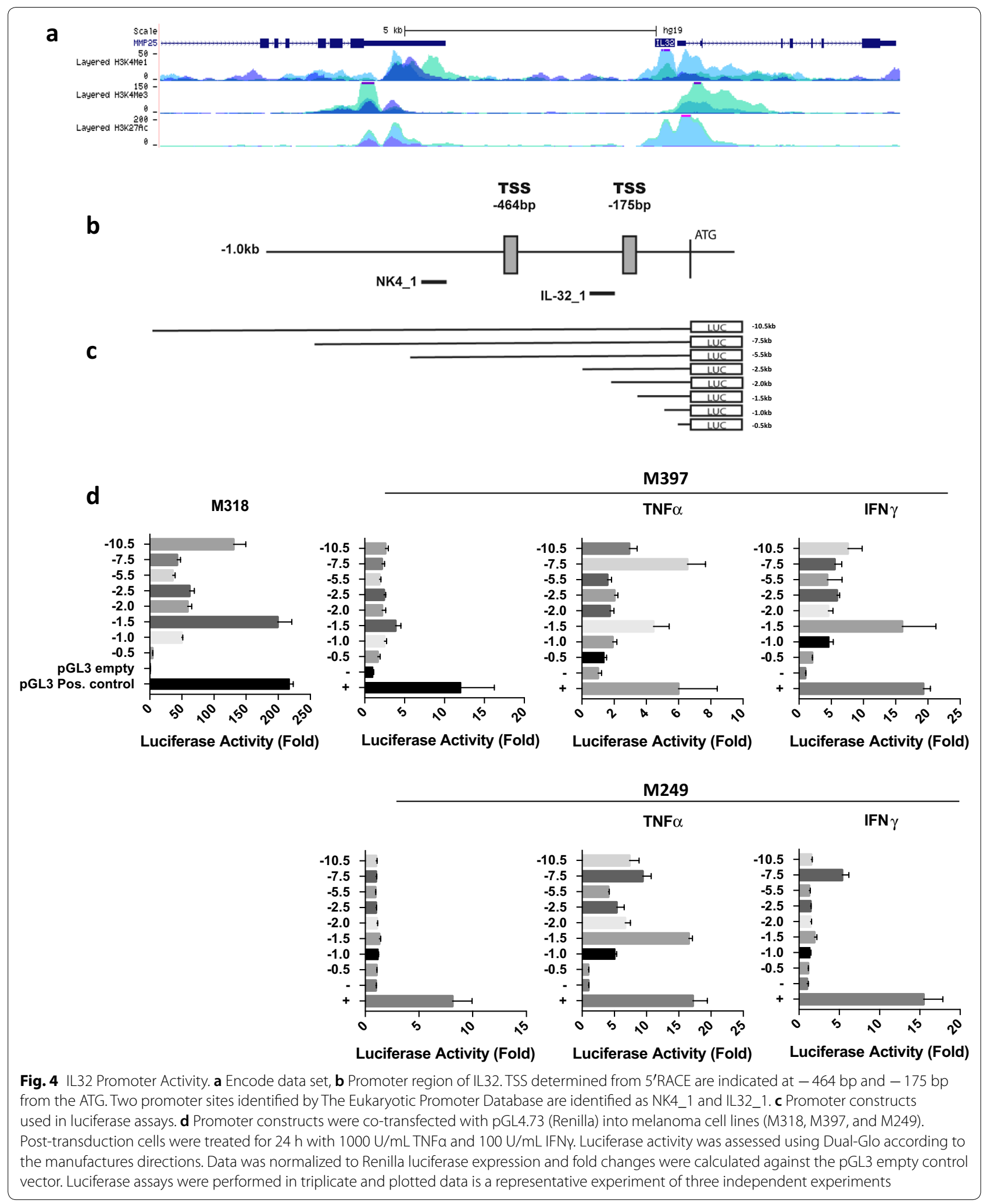


tumor biopsies as part of The Cancer Genome Atlas (TCGA) dataset. We observed strong Pearson correlation coefficients between IL32 expression, found within the melanoma tumor and the surrounding microenvironment, and genes related to immune cell infiltration, such as CD3E (0.94), CD8A (0.89), IFNY (0.75), and GZMB (0.86) (Additional file 3: Figure S3). Additionally, we observed a strong correlation between IL32 expression and several checkpoint receptors and ligands, such as PD-1 (0.87), PD-L1 (0.69), Tim-3 (0.82), Lag3 (0.85), and TIGIT (0.86) (Additional file 4: Figure S4). We also observed correlations between IL32 and genes related to other immune subsets, including myeloid cells (ITGAM, 0.71; CD68, 0.61; CD14, 0.74), dendritic cells (ITGAX, 0.71; ITGAE, 0.47) and B cells (CD19, 0.67). Consistent with this, the top 100 genes that correlated with IL32 expression in the TCGA dataset were enriched with gene sets related to allograft rejection (hypergeometric $\mathrm{p}$-value $=4.3 \times 10^{-36}$ ), inflammatory response (hypergeometric $\mathrm{p}$-value $=3.3 \times 10^{-10}$ ), and IFN $\gamma$ signaling (hypergeometric $\mathrm{p}$-value $=8.3 \times 10^{-9}$ ).

In light of the impact of a proinflammatory microenvironment on melanoma differentiation, as well as the association between IL32 and dedifferentiation in melanoma cell lines, we investigated if this phenomenon was also present in the TCGA dataset. We observed a correlation between IL32 expression in melanoma tumor biopsies with a high AXL/low MITF drug-resistant signature, consistent with data from melanoma cell lines (Additional file 3: Figure S3). However, IL32 did not inversely correlate with signature melanocyte genes (MLANA, $P M E L, T Y R$ ) as we observed in the cell line data (data not shown). Additionally, IL32 expression did not correlate with genes associated with disease progression, tumor cell invasion, and migration, such as CD155/PRV and MMP2 (Additional file 4: Figure S4).

\section{IL32 isoforms have differential impact on myeloid polarization}

IL32 from melanoma cells may influence non-melanoma cells in the tumor microenvironment, such as myeloid cells, which can play both pro- and anti-tumor roles depending on their phenotypic state. We preliminarily assessed of the impact of IL32 on the polarization of myeloid cells. It has previously been reported that IL32 $\gamma$ induces the differentiation of monocytes into phagocytic macrophage-like cells (though they discordantly express CD14 and CD1a) [19]. However, the effect of IL32 $\gamma$, or other IL32 isoforms (IL32 $\alpha$ and IL32 $\beta$ ), on myeloid cell polarization is not well understood. We observed that monocytes cultured with either IL32 $\beta$ or IL32 $\gamma$ express both CD68 and CD80, markers associated with M1 proinflammatory macrophages thought to play an antitumor role (Additional file 5: Figure S5). In contrast, exposure to IL-32 $\alpha$ resulted in a CD68+ but CD80population. Likewise, IL32 $\beta$ and IL32 $\gamma$, but not IL32 $\alpha$, resulted in higher CD14 and CD1b expression.

\section{Discussion}

Human melanomas produce a number of cytokines generally associated with the immune system [38]. In an analysis of the RNA transcripts from 53 established melanoma lines, a significant proportion express IL32 isoforms. Thus, we evaluated the biology underlying IL32 expression in the context of melanoma both in cell lines and in melanoma tumor samples.

IL32 is found on human chromosome 16p13.3, is expressed in at least 6 splice variants $(\alpha, \beta, \gamma, \delta, \varepsilon, \zeta)$ and was originally isolated from activated natural killer and $\mathrm{T}$ cells [16]. Which isoforms are secreted as opposed to acting intracellularly is still unresolved and hampered by an as of yet unidentified cell surface receptor $[39,40]$. The $\gamma$ isoform is the only isoform that has a leader sequence, is composed of all exons, is believed to be secreted, and believed to be the most biologically active [41]. A rodent IL32 homologue does not exist-raising the obvious question as to whether or not this gene product is dispensable or redundant-but human recombinant and transgenic IL32 appears to be biologically active in mice [12, 42-44]. Despite these gaps in our knowledge, much of the published literature on the biology of IL32 comes from experiments utilizing commercially available recombinant protein, generally the $\alpha, \beta$, and $\gamma$ isoforms [1].

Several themes emerge regarding IL32 biology: (a) IL32 can modulate the production of various cytokines including IL1 $\beta$ [45], TNF $\alpha$ [46], IL6 [47], IL8 [8], and may activate cells of the immune system [15, 19]; (b) IL32 expression can be induced by various viruses and microbes $[2,20,42,48-51]$ and may play a role in antiviral immunity [52]; (c) IL32 may have direct effects on various cancer cells in vitro; [8, 9, 22, 47, 53, 54]; (d) IL32 may play a role in tumor immunity $[4,7,12,14,44]$; [41]; and (e) IL32 has been associated with various inflammatory diseases $[43,45]$, such as ulcerative colitis and rheumatoid arthritis [55]. It is also curious that, largely from immunohistochemistry studies, IL32 is expressed by a broad range of epithelial and in hematopoietic malignancies and is generally correlated with aggressive biology, although no unifying mechanism has been identified.

In comparing IL32 expressing and non-expressing melanoma cell lines, we observed that IL32 expression in melanoma cell lines correlates with a high AXL/ low MITF ratio, a genetic signature which has been reported to be associated with a treatment-resistant, dedifferentiation "invasive" phenotype. This dedifferentiation 
phenotype results in reduced expression of melanocyticlineage antigens, which may also provide a mechanism for immune evasion [32]. Melanomas, which progress on MAPK inhibitor therapies, acquire this genetic signature of reduced MITF and RTK upregulation [25]. MITF controls the expression of a broad range of genes in melanocyte-lineage cells that govern differentiation, migration, and proliferation [56-58]. The low MITF signature is regulated by receptor tyrosine kinases, including AXL [59].

The plasticity of these neural crest-derived cutaneous malignancies is underscored by the ability of an inflammatory signal-TNF $\alpha$ or IFN $\gamma$, for example-to effect a differentiated to dedifferentiated conversion in melanoma cell lines with only a $72 \mathrm{~h}$ in vitro exposure, a phenomenon that reverses when the cytokine is removed from culture [26]. We found that TNF $\alpha$ and IFN $\gamma$, which promote a dedifferentiated melanoma phenotype, also induce IL32 expression in non-IL32 expressing melanoma cell lines by impacting activity at the promoter level.

Palstra et al. recently reported that the DNA element encompassing rs4349147 is a strong long distance enhancer essential for the expression of IL32 in CD4 $\mathrm{T}$ cells located $10 \mathrm{~kb} 3^{\prime}$ of the IL32 promoter [52]. Jurkat cells are heterozygous for the rs4349147-A and rs4349147-G alleles. The A allele increases the expression of IL32 $\alpha$, generally viewed as anti-inflammatory, whereas the $\mathrm{G}$ allele promoted the expression of the proinflammatory IL32 $\gamma$ and IL32 $\beta$ isoforms. These proinflammatory isoforms enhanced lymphocyte activation and susceptibility to HIV infection. This report provides considerable insight into various observations on the biology of IL32 isoforms. Two putative IL32 promoters have been identified using the Eukaryotic Promoter Database (http://epd. vital-it.ch), which are depicted in Fig. 4. These two promoters, at least in Jurkat cells, designated EPD NK4_1 and EPD IL32_1, are thought to support the transcription of IL32 $\gamma$ and IL32 $\alpha / \beta$, respectively.

Our IL32 promoter constructs encompass both the identified EPD NK4_1 and EPD IL32_1 putative promoter regions and extend $10.5 \mathrm{~kb} 5^{\prime}$ to the ATG. The luciferase activity driven by these constructs would then necessarily reflect constitutive and induced gene expression of all three isoforms, as well as the putative cis-acting elements residing within this $5^{\prime}$ upstream region. Palstra et al. performed formaldehyde-assisted isolation of regulatory elements (FAIRE) assays in Jurkat and a melanoma cell line (G361), which demonstrated significant increase in DNA accessibility in the region surrounding rs4349147 in the former but not the latter cell line [52]. We therefore conjecture that this long-range enhancer may not play a role in melanoma IL32 transcription. In another IL32 promoter study using Akt-activated endothelial cell line constructs extending $2.5 \mathrm{~kb} 5^{\prime}$ to the ATG [60]; the difference in cell lines and activation signals precludes any meaningful comparison.

Given the impact of proinflammatory cytokines on IL32 expression, we investigated the IL32 expression in immunologically rich melanoma tumors using RNA sequencing data available in the TCGA dataset. IL32 expression was highly correlated with a $\mathrm{T}$ cell dominant immune signature in these tumors, probably a result of immune cell IL32 production. We also observed a correlation between IL32 expression, derived from tumor cells and the surrounding microenvironment, and a high AXL/ low MITF gene signature, as in the melanoma cell lines. However, we interpret this result cautiously because, unlike in the melanoma cell line dataset, IL32 expression in the melanoma tumor microenvironment did not inversely correlate with pigmentation or melanoma differentiation genes such as MLANA, TYR or PMEL. Thus, the high AXL/low MITF signature may be a result of increased IL32 expression by infiltrating immune cells, rather than IL32 expressing dedifferentiated melanoma cells in the tumor microenvironment.

There is substantial evidence that IL32 induces dedifferentiation of human monocytes towards a macrophagelike phonotype with dendritic cell-like aspects. IL32 exposed monocytes altered their morphology within 3 days (flattening with extensive pseudopodia), which was accompanied by increased expression of CD1 and CD14 [19]. These macrophage-like cells exhibited active phagocytic properties and were also induced to express TNF $\alpha$, IL-1 $\beta$ and IL6. Our preliminary in vitro studies indicated that IL32, in an IL32 $\beta$ and IL32 $\gamma$ isoform specific manner, can modulate the polarization of myeloid cells toward an M1-like macrophage phenotype with costimulatory molecule expression, lending additional support for its impact in the tumor microenvironment. It is plausible, then, that IL32 is expanded within the microenvironment as a byproduct of the feedback loop between the antitumor inflammatory response (marked by TNF $\alpha$ and IFNY) and dedifferentiation of melanoma [23].

\section{Conclusions}

In summary, this study shows that a significant percentage of cell lines derived from metastatic melanoma express IL32. IL32 expression, which correlates with a dedifferentiated "invasive" genetic signature, can be induced by inflammatory molecules TNF $\alpha$ and IFN $\gamma$ that modulate IL32 transcriptional activity at the promoter level. IL32 expression by dedifferentiated tumor cells may contribute to a proinflammatory tumor microenvironment.

The remarkable advances in melanoma immunotherapy occasioned by the development of CTLA-4 
and PD-1/PDL1 checkpoint inhibition underscores the importance of understanding potential contributions of melanoma-elaborated cytokines such as IL32 [61].

\section{Additional files}

Additional file 1: Figure S1. Expression of IL32 in human melanoma. (A) Immunohistochemistry analysis of IL32 expression in cutaneous melanoma in two patients with low and high IL32 expression, respectively (images and data acquired from The Human Protein Atlas, and Image available at https://www.proteinatlas.org/ENSG00000008517-IL32/patho logy/tissue/melanoma\#img). Left panel is a cutaneous melanoma sample from a 73-year old woman (patient id: 2900). Right panel is a cutaneous melanoma sample from an 83-year old man (patient id: 2156). Staining was performed using BioLegend mouse anti-human IL32 monoclonal antibody (Cat \# 513401) at a 1:4500 dilution after HIER antigen retrieval ( $\mathrm{pH}=6$ ). ( $\mathrm{B}$ and $\mathrm{C}$ ) IL32 transcript expression across multiple different cancer lines organized by cancer type from the NCl-60 Cancer Cell Line database and the Cancer Cell Line Encyclopedia, respectively.

Additional file 2: Figure S2. Addition of recombinant IL32 does not impact melanoma cell line growth or differentiation. (A) Tumor growth over time in parental M397 melanoma cell line, compared to M397 treated with recombinant IL $32 a,-\beta$ or $-\gamma$. (B and C) Expression of melanoma differentiation genes by quantitative RT-PCR at baseline or after treatment with TNFa, recombinant IL32a, IL32 $\beta$, or IL32 $\gamma$ measured at day 3 (B) or day 7 (C).

Additional file 3: Figure S3. IL32 expression in the TCGA dataset. (A-B) Scatterplot of log2 FPKM expression values between IL32 and select immune genes (A) or between the ratio of AXL and MITF log2 FPKM expression values (B) in the melanoma TCGA dataset $(n=479)$.

Additional file 4: Figure S4. IL32, checkpoint receptors/ligand expression, and markers of disease progression in the TCGA dataset. Scatterplot of log2 FPKM expression values between IL32 and select checkpoint receptors/ligands, as well as, select markers of disease progression (MMP2 and PVR).

Additional file 5: Figure S5. Expression of phenotypic markers on human monocytes after exposure to stimuli. CD14+cells, from PBMC isolation, were cultured in the presence of GM-CSF + IL-4, Cell Genix Media, recIL32a, recIL32 $\beta$, or recIL32 $\gamma$ for 5 days. The different treatments are displayed by using a gray scale. On day 5 , the phenotype of the cells was assayed using flow cytometry analysis for various surface markers. GM-CSF + IL-4 and Cell Genix Media alone were as a positive and negative control, respectively. Cells were then assessed for surface expression of CD68, CD80, CD14, CD1B by flow cytometry. Frequency of positive cells is shown in the left panels, and mean fluorescence intensity is shown in the right panels. Statistical analysis was done using a one-way repeated measures ANOVA, using the GM-CSF + IL-4 treatment as a control. Each shape (circle, square, triangle) represents a different healthy donor. ${ }^{*} \mathrm{P} \leq 0.05$, ${ }^{* *} \mathrm{P} \leq 0.01$, ***P $\leq 0.001$.

\section{Abbreviations}

IL32: interleukin 32; TCGA: the cancer genome atlas; ATG: translational start site; RACE: rapid amplification of cDNA ends.

\section{Authors' contributions}

HP performed, analyzed, and interpreted the human cell line and IL32 promoter data. JT analyzed and interpreted the sequencing and TCGA data. AK analyzed and interpreted the TCGA data. CSG analyzed the sequencing and TCGA data. DMM performed, analyzed and interpreted the myeloid cell data. WHM, DS, LHB, AR, TGG, and JSE all contributed to the design of experiments and the interpretation of results. HP, JT, AK, DMM and JSE were significantly involved in writing of this manuscript. All authors read and approved the final manuscript.

\section{Author details}

${ }^{1}$ Department of Surgery, University of California, Los Angeles, 10833 Le Conte Ave, Los Angeles, CA 90095, USA. ${ }^{2}$ Department of Microbiology, Immunology, and Molecular Genetics, University of California, Los Angeles, CA 90095, USA. ${ }^{3}$ Department of Molecular and Medical Pharmacology, Crump Institute for Molecular Imaging, University of California, Los Angeles, CA 90095, USA. ${ }^{4}$ Department of Radiation Oncology, David Geffen School of Medicine, University of California, Los Angeles, CA 90095, USA. ${ }^{5}$ Department of Medicine, David Geffen School of Medicine, University of California, Los Angeles, CA 90095, USA. ${ }^{6}$ Jonsson Comprehensive Cancer Center, University of California, Los Angeles, CA 90095, USA. ${ }^{7}$ Department of Immunology, University of Pittsburgh, Pittsburgh, PA 15213, USA. ${ }^{8}$ Department of Medicine, University of Pittsburgh Cancer Institute, Pittsburgh, PA 15213, USA. ${ }^{9}$ Department of Surgery, University of Pittsburgh Cancer Institute, Pittsburgh, PA 15213, USA. ${ }^{10}$ Department of Clinical and Translational Science, University of Pittsburgh, Pittsburgh, PA 15213, USA.

\section{Acknowledgements}

We appreciate constructive input from Dr. Michael Carey (UCLA).

\section{Competing interests}

LHB declares Simpatica, Scientific Advisory Board Member, Jan. 2017-present; Stemlmmune Scientific and Medical Advisory Board, April 6, 2017-present; SapVax Advisory Board Nov. 15, 2017-present; NextCure, Scientific Advisory Board, 2018-present; Replimmune, Scientific Advisory Board, 2018-present; Western Oncolytics, Scientific Advisory Board, 2018-present; Torque Therapeutics, Consultant, 2018-present. AR has received honoraria from consulting with Bristol Myers-Squibb, Amgen, Chugai, Genentech, Merck, Novartis and Roche, and is on the scientific advisory board of Advaxis, Arcus, Bioncotech, Compugen, CytomX, Five Prime, FLX-Bio, ImaginAb, Isoplexis, Merus and Rgenix. During the conduct of this work AR was on the scientific advisory board and held stock in Kite-Pharma, and is co-founder of PACT Pharma and Tango Therapeutics. JSE is a scientific advisor to Allogene Therapeutics and Neogene Therapeutics.

\section{Availability of data and materials}

One of the datasets analyzed during the current study is included in the Gene Expression Omnibus (GEO), https://www.ncbi.nlm.nih.gov/geo/query/acc. cgi?acc $=$ GSE80829 [26]. Additional datasets analyzed in this current study are available from The Cancer Genome Atlas, https://cancergenome.nih.gov/.

\section{Consent for publication}

Not applicable.

Ethics approval and consent to participate

Not applicable.

\section{Funding}

Univ. Pittsburgh Skin Biology T32 Training Grant pre-doctoral Scholar (DMM); P50 CA121973-03 Pittsburgh SPORE in Melanoma and Skin Cancer (LHB); NIH NCI PO1 DCA 168585 (AR, TGG); NIH 1R01CA191234-01A1 (DS); Joy and Jerry Monkarsh Fund (JSE); Vincent Price Research Fund (JSE).

\section{Publisher's Note}

Springer Nature remains neutral with regard to jurisdictional claims in published maps and institutional affiliations.

Received: 18 January 2019 Accepted: 27 March 2019

Published online: 05 April 2019

\section{References}

1. Heinhuis B, Netea MG, van den Berg WB, Dinarello CA, Joosten LA. Interleukin-32: a predominantly intracellular proinflammatory mediator that controls cell activation and cell death. Cytokine. 2012;60(2):321-7.

2. Joosten $L A$, Heinhuis $B$, Netea MG, Dinarello CA. Novel insights into the biology of interleukin-32. Cell Mol Life Sci. 2013;70(20):3883-92. 
3. Khawar MB, Abbasi MH, Sheikh N. IL-32: a novel pluripotent inflammatory interleukin, towards gastric inflammation, gastric cancer, and chronic rhino sinusitis. Mediat Inflamm. 2016;2016:8413768.

4. Wang S, Chen F, Tang L. IL-32 promotes breast cancer cell growth and invasiveness. Oncol Lett. 2015;9(1):305-7.

5. Wang Y, Yang Y, Zhu Y, Li L, Chen F, Zhang L. Polymorphisms and expression of IL-32: impact on genetic susceptibility and clinical outcome of lung cancer. Biomarkers. 2017;22(2):165-70.

6. Yang Y, Wang Z, Zhou Y, Wang X, Xiang J, Chen Z. Dysregulation of overexpressed IL-32 in colorectal cancer induces metastasis. World J Surg Oncol. 2015;13:146

7. Oh JH, Cho MC, Kim JH, Lee SY, Kim HJ, Park ES, et al. IL-32gamma inhibits cancer cell growth through inactivation of NF-kappaB and STAT3 signals. Oncogene. 2011;30(30):3345-59.

8. Park ES, Yoo JM, Yoo HS, Yoon DY, Yun YP, Hong J. IL-32gamma enhances TNF-alpha-induced cell death in colon cancer. Mol Carcinog. 2014;53(Suppl 1):E23-35.

9. Yun HM, Park KR, Kim EC, Han SB, Yoon DY, Hong JT. IL-32alpha suppresses colorectal cancer development via TNFR1-mediated death signaling. Oncotarget. 2015:6(11):9061-72.

10. Nishida A, Andoh A, Inatomi O, Fujiyama Y. Interleukin-32 expression in the pancreas. J Biol Chem. 2009;284(26):17868-76.

11. Plantinga TS, Costantini I, Heinhuis B, Huijbers A, Semango G, Kusters B, et al. A promoter polymorphism in human interleukin-32 modulates its expression and influences the risk and the outcome of epithelial cellderived thyroid carcinoma. Carcinogenesis. 2013;34(7):1529-35.

12. Heinhuis B, Plantinga TS, Semango G, Kusters B, Netea MG, Dinarello CA et al. Alternatively spliced isoforms of IL-32 differentially influence cell death pathways in cancer cell lines. Carcinogenesis. 2016;37(2):197-205.

13. Gorvel L, Korenfeld D, Tung T, Klechevsky E. Dendritic cell-derived IL-32alpha: a novel inhibitory cytokine of NK cell function. J Immunol. 2017;199(4):1290-300.

14. Cheon S, Lee JH, Park S, Bang SI, Lee WJ, Yoon DY, et al. Overexpression of IL-32alpha increases natural killer cell-mediated killing through up-regulation of Fas and UL16-binding protein 2 (ULBP2) expression in human chronic myeloid leukemia cells. J Biol Chem. 2011;286(14):12049-55.

15. Park MH, Song MJ, Cho MC, Moon DC, Yoon DY, Han SB, et al. Interleukin-32 enhances cytotoxic effect of natural killer cells to cancer cells via activation of death receptor 3. Immunology. 2012;135(1):63-72.

16. Kim SH, Han SY, Azam T, Yoon DY, Dinarello CA. Interleukin-32: a cytokine and inducer of TNFalpha. Immunity. 2005;22(1):131-42.

17. Shoda H, Fujio K, Yamaguchi Y, Okamoto A, Sawada T, Kochi Y, et al. Interactions between IL-32 and tumor necrosis factor alpha contribute to the exacerbation of immune-inflammatory diseases. Arthritis Res Ther. 2006;8(6):R166

18. Li W, Liu Y, Mukhtar MM, Gong R, Pan Y, Rasool ST, et al. Activation of interleukin-32 pro-inflammatory pathway in response to influenza A virus infection. PLOS ONE. 2008;3(4):e1985.

19. Netea MG, Lewis EC, Azam T, Joosten LA, Jaekal J, Bae SY, et al. Interleukin-32 induces the differentiation of monocytes into macrophage-like cells. Proc Natl Acad Sci USA. 2008;105(9):3515-20.

20. Ohmatsu H, Humme D, Gonzalez J, Gulati N, Mobs M, Sterry W, et al. IL-32 induces indoleamine 2,3-dioxygenase $(+) \mathrm{CD} 1 \mathrm{C}(+)$ dendritic cells and indoleamine 2,3-dioxygenase(+)CD163(+) macrophages: relevance to mycosis fungoides progression. Oncoimmunology. 2017:6(2):e1181237.

21. Suga H, Sugaya M, Miyagaki T, Kawaguchi M, Fujita H, Asano Y, et al. The role of IL-32 in cutaneous T-cell lymphoma. J Invest Dermatol. 2014;134(5):1428-35.

22. Lee J, Kim KE, Cheon S, Song JH, Houh Y, Kim TS, et al. Interleukin-32alpha induces migration of human melanoma cells through downregulation of E-cadherin. Oncotarget. 2016;7(40):65825-36.

23. Taube JM, Young GD, McMiller TL, Chen S, Salas JT, Pritchard TS, et al. Differential expression of immune-regulatory genes associated with PD-L1 display in melanoma: implications for PD-1 pathway blockade. Clin Cancer Res. 2015;21 (17):3969-76.

24. Konieczkowski DJ, Johannessen CM, Abudayyeh O, Kim JW, Cooper ZA, Piris $A$, et al. A melanoma cell state distinction influences sensitivity to MAPK pathway inhibitors. Cancer Discov. 2014;4(7):816-27.

25. Muller J, Krijgsman O, Tsoi J, Robert L, Hugo W, Song C, et al. Low MITF/ AXL ratio predicts early resistance to multiple targeted drugs in melanoma. Nat Commun. 2014:5:5712.
26. Tsoi J, Robert L, Paraiso K, Galvan C, Sheu KM, Lay J, et al. Multi-stage differentiation defines melanoma subtypes with differential vulnerability to drug-induced iron-dependent oxidative stress. Cancer Cell. 2018;33(5):890-904

27. Landsberg J, Kohlmeyer J, Renn M, Bald T, Rogava M, Cron M, et al. Melanomas resist T-cell therapy through inflammation-induced reversible dedifferentiation. Nature. 2012:490(7420):412-6.

28. Uhlen M, Bjorling E, Agaton C, Szigyarto CA, Amini B, Andersen E, et al. A human protein atlas for normal and cancer tissues based on antibody proteomics. Mol Cell Proteom. 2005;4(12):1920-32.

29. Uhlen M, Zhang C, Lee S, Sjostedt E, Fagerberg L, Bidkhori G, et al. A pathology atlas of the human cancer transcriptome. Science. 2017:357:6352

30. Cerami E, Gao J, Dogrusoz U, Gross BE, Sumer SO, Aksoy BA, et al. The cBio cancer genomics portal: an open platform for exploring multidimensional cancer genomics data. Cancer Discov. 2012;2(5):401-4.

31. Gao J, Aksoy BA, Dogrusoz U, Dresdner G, Gross B, Sumer SO, et al. Integrative analysis of complex cancer genomics and clinical profiles using the cBioPortal. Sci Signal. 2013;6(269):pl1.

32. Mehta A, Kim YJ, Robert L, Tsoi J, Comin-Anduix B, Berent-Maoz B, et al. Immunotherapy resistance by inflammation-induced dedifferentiation. Cancer Discov. 2018;8(8):935-43.

33. Liberzon A, Birger C, Thorvaldsdottir H, Ghandi M, Mesirov JP, Tamayo P. The molecular signatures database (MSigDB) hallmark gene set collection. Cell Syst. 2015;1 (6):417-25.

34. Guenther MG, Levine SS, Boyer LA, Jaenisch R, Young RA. A chromatin landmark and transcription initiation at most promoters in human cells. Cell. 2007;130(1):77-88.

35. Zhang T, Cooper S, Brockdorff N. The interplay of histone modificationswriters that read. EMBO Rep. 2015;16(11):1467-81.

36. Zhou VW, Goren A, Bernstein BE. Charting histone modifications and the functional organization of mammalian genomes. Nat Rev Genet. 2011;12(1):7-18.

37. Soares LM, He PC, Chun Y, Suh H, Kim T, Buratowski S. Determinants of histone H3K4 methylation patterns. Mol Cell. 2017;68(4):773-85.

38. Qin Y, Milton DR, Oba J, Ding Z, Lizee G, Ekmekcioglu S, et al. Inflammatory IL-1 beta-driven JNK activation in stage III melanoma. Pigment Cell Melanoma Res. 2015;28(2):236-9.

39. Heinhuis B, Koenders MI, van den Berg WB, Netea MG, Dinarello CA, Joosten LA. Interleukin 32 (IL-32) contains a typical alpha-helix bundle structure that resembles focal adhesion targeting region of focal adhesion kinase-1. J Biol Chem. 2012;287(8):5733-43.

40. Novick D, Rubinstein M, Azam T, Rabinkov A, Dinarello CA, Kim SH. Proteinase 3 is an IL-32 binding protein. Proc Natl Acad Sci USA. 2006;103(9):3316-21.

41. Choi JD, Bae SY, Hong JW, Azam T, Dinarello CA, Her E, et al. Identification of the most active interleukin-32 isoform. Immunology. 2009;126(4):535-42.

42. Bai X, Shang S, Henao-Tamayo M, Basaraba RJ, Ovrutsky AR, Matsuda $\mathrm{JL}$, et al. Human IL-32 expression protects mice against a hypervirulent strain of Mycobacterium tuberculosis. Proc Natl Acad Sci USA. 2015;112(16):5111-6

43. Kim SJ, Lee S, Kwak A, Kim E, Jo S, Bae S, et al. Interleukin-32gamma transgenic mice resist LPS-mediated septic shock. J Microbiol Biotechnol. 2014;24(8):1133-42.

44. Qu Y, Taylor JL, Bose A, Storkus WJ. Therapeutic effectiveness of intratumorally delivered dendritic cells engineered to express the pro-inflammatory cytokine, interleukin (IL)-32. Cancer Gene Ther. 2011;18(9):663-73.

45. Khawar B, Abbasi MH, Sheikh N. A panoramic spectrum of complex interplay between the immune system and IL-32 during pathogenesis of various systemic infections and inflammation. Eur J Med Res. 2015;20:7.

46. Kim MS, Kang JW, Jeon JS, Kim JK, Kim JW, Hong J, et al. IL-32theta gene expression in acute myeloid leukemia suppresses TNF-alpha production. Oncotarget. 2015;6(38):40747-61.

47. Kang JW, Park YS, Lee DH, Kim JH, Kim MS, Bak Y, et al. Intracellular interaction of interleukin (IL)-32alpha with protein kinase Cepsilon (PKCepsilon) and STAT3 protein augments IL- 6 production in THP-1 promonocytic cells. J Biol Chem. 2012;287(42):35556-64.

48. Dos Santos JC, Heinhuis B, Gomes RS, Damen MS, Real F, Mortara RA, et al. Cytokines and microbicidal molecules regulated by IL-32 in 
THP-1-derived human macrophages infected with New World Leishmania species. PLoS Negl Trop Dis. 2017;1 1(2):e0005413.

49. Montoya D, Inkeles MS, Liu PT, Realegeno S, Teles RM, Vaidya P, et al. IL-32 is a molecular marker of a host defense network in human tuberculosis. Sci Transl Med. 2014;6(250):250ra114.

50. Schenk M, Krutzik SR, Sieling PA, Lee DJ, Teles RM, Ochoa MT, et al. NOD2 triggers an interleukin-32-dependent human dendritic cell program in leprosy. Nat Med. 2012;18(4):555-63.

51. Zhou Y, Zhu Y. Important role of the IL-32 inflammatory network in the host response against viral infection. Viruses. 2015;7(6):3116-29.

52. Palstra RJ, de Crignis E, Roling MD, van Staveren T, Kan TW, van ljcken W, et al. Allele-specific long-distance regulation dictates IL-32 isoform switching and mediates susceptibility to HIV-1. Sci Adv. 2018;4(2):e1701729.

53. Nicholl MB, Chen X, Qin C, Bai Q, Zhu Z, Davis MR, et al. IL-32alpha has differential effects on proliferation and apoptosis of human melanoma cell lines. J Surg Oncol. 2016;113(4):364-9.

54. Yun HM, Oh JH, Shim JH, Ban JO, Park KR, Kim JH, et al. Antitumor activity of IL-32 beta through the activation of lymphocytes, and the inactivation of NF-kappaB and STAT3 signals. Cell Death Dis. 2013;4:e640.
55. Heinhuis B, Koenders MI, van de Loo FA, Netea MG, van den Berg WB, Joosten LA. Inflammation-dependent secretion and splicing of IL-32\{gamma\} in rheumatoid arthritis. Proc Natl Acad Sci USA. 2011;108(12):4962-7.

56. Hartman ML, Czyz M. MITF in melanoma: mechanisms behind its expression and activity. Cell Mol Life Sci. 2015;72(7):1249-60.

57. Yajima I, Kumasaka MY, Thang ND, Goto Y, Takeda K, lida M, et al. Molecular network associated with MITF in skin melanoma development and progression. J Skin Cancer. 2011;2011:730170.

58. Hsiao JJ, Fisher DE. The roles of microphthalmia-associated transcription factor and pigmentation in melanoma. Arch Biochem Biophys. 2014;563:28-34.

59. Ahmed F, Haass NK. Microenvironment-driven dynamic heterogeneity and phenotypic plasticity as a mechanism of melanoma therapy resistance. Front Oncol. 2018;8:173.

60. Kobayashi H, Lin PC. Molecular characterization of IL-32 in human endothelial cells. Cytokine. 2009;46(3):351-8.

61. Lugowska I, Teterycz P, Rutkowski P. Immunotherapy of melanoma. Contemp Oncol (Pozn). 2018;22(1A):61-7.
Ready to submit your research? Choose BMC and benefit from:

- fast, convenient online submission

- thorough peer review by experienced researchers in your field

- rapid publication on acceptance

- support for research data, including large and complex data types

- gold Open Access which fosters wider collaboration and increased citations

- maximum visibility for your research: over 100M website views per year

At BMC, research is always in progress.

Learn more biomedcentral.com/submissions 